\title{
Harnessing the developmental potential of nucellar cells: barriers and opportunities
}

\author{
R.M. Ranganath \\ Cytogenetics and Developmental Biology Laboratory, Department of Botany, Bangalore University, Jnanabharathi Campus, \\ Bangalore 560056, India
}

\begin{abstract}
Angiosperm nucellar cells can either use or avoid meiosis in vivo, depending on the developmental context. This unique ability contrasts with the conditions required in vitro, either for a reconstituted oocyte to avoid meiosis and produce clones by somatic cell nuclear transfer (SCNT), or for mammalian stem cells to undergo meiosis and produce synthetic sex cells (gametes). Current biotechnological initiatives to harness the potential of nucellar cells are based on the transfer of apomixis genes to sexual crop plants with the aim of producing clones through seeds. The elusive genetic basis of apomixis compels us to examine whether this process involves epigenetic factors. The elegant and versatile developmental platform available in nucellar cells should be explored as a genome-scale science and compared with mammalian stem cell biology for a holistic understanding of developmental programming and reprogramming in eukaryotes.
\end{abstract}

Recent initiatives for cloning animals by somatic cell nuclear transfer (SCNT) and for in vitro gamete production have propelled meiosis to several new areas of relevance in biology. However, the focus has now shifted from what happens during meiosis to creating appropriate in vitro conditions in which the stem cells or reconstituted oocytes can use or avoid meiosis to generate a desired developmental output. By contrast, angiosperm nucellar cells possess the ability to use or to avoid meiosis in vivo without compromising further development. A nucellar cell can also transdifferentiate directly into an adventitious embryo. The in vitro mammalian stem cell system and the nucellar cell development in planta are powerful experimental models that have relevance both to the basic biology of developmental programming and/or reprogramming and to biotechnological opportunities.

Here I argue that the novel features and challenges in nucellar cell biology are as exciting as those in mammalian stem cell biology and deserve due recognition by cell biologists for adoption and promotion as a genome-scale science. In addition, I argue that a molecular definition of nucellar cell development will provide much-needed

Corresponding author: R.M. Ranganath (rayasran@rediffmail.com). insights into ongoing biotechnological initiatives based on genetic manipulations of apomixis.

\section{Synthetic gametes and SCNT: to use or to avoid meiosis?}

Current mammalian stem cell research revolves around the competence of in vitro technologies either to induce or to avoid meiosis. Although animals have dedicated germline stem cells for producing gametes, the in vitro production of gametes was not achieved until Hubner et al. [1] and Geijsen et al. [2] reached this landmark. The synthetic production of gametes hinges on the ability of the stem cell to undergo meiosis and maturation under in vitro conditions.

By contrast, the technological challenges involved in cloning by SCNT hinge on the competence of the reconstituted oocyte - a somatic cell nucleus fused with an enucleated oocyte - to sidestep meiosis (haploidy) and fertilization, and to jumpstart development to an embryonic state. Three effective strategies have been developed, by coordinating the cell cycles of the recipient oocyte and the donor nucleus, to coax the reconstituted oocyte into behaving like a diploid cell instead of producing haploid cells by meiosis [3]. In the Honolulu cloning technique, for example, Cytochalasin B is used specifically to force the reconstituted oocyte to sidestep meiosis (to suppress polar body formation) and to maintain the donor somatic nucleus in a diploid condition necessary for embryonic development (Figure 1).

Nucellus: a tissue in search of a developmental identity Angiosperm nucellar cells rival the charismatic developmental and biotechnological potential of mammalian stem cells. The nucellus is a morphologically homogeneous ovular tissue. Normally, a nucellar cell (which does not self-renew like a stem cell) that is committed to development behaves as a progenitor cell and kick starts a highly conserved developmental pathway involving meiosis to produce a female gametophyte (FG) with a haploid egg. This fidelity can be relaxed in two distinct forms of apomictic pathways in angiosperms: the gametophytic (apospory or diplospory) pathway, and the sporophytic (adventitious embryony) pathway.

In the first pathway, either the megaspore mother cell (MMC), which differentiates from the nucellar cells, might 
(a)

(b)

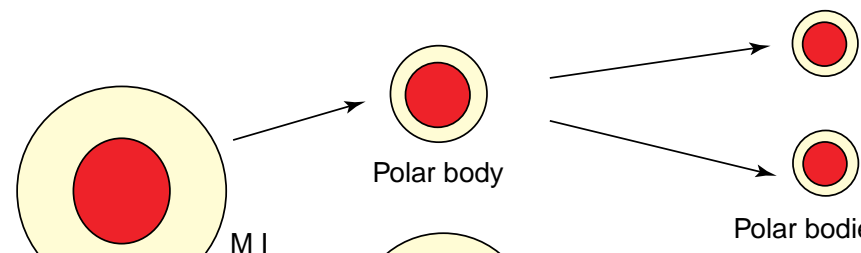

Primary oocyte

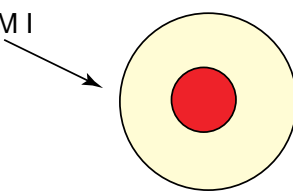

Secondary oocyte
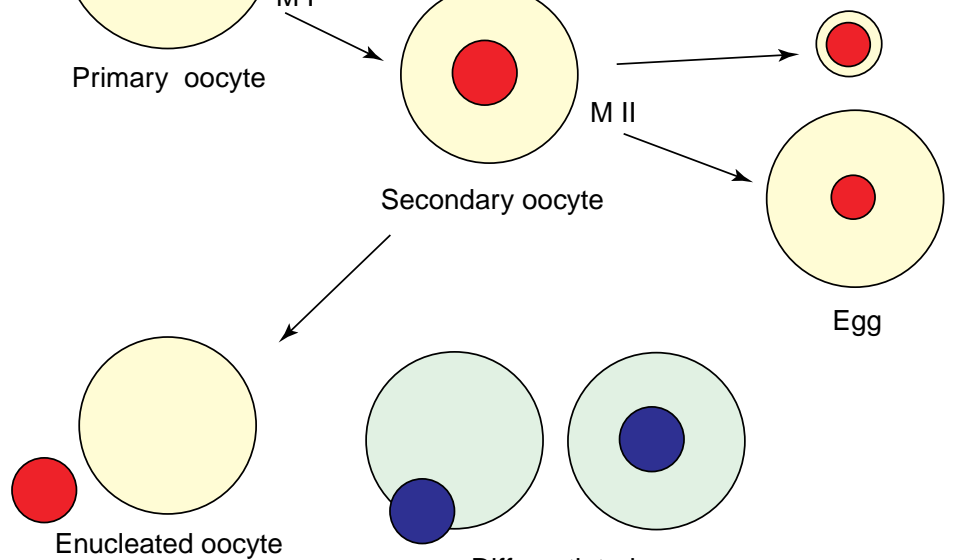

Egg
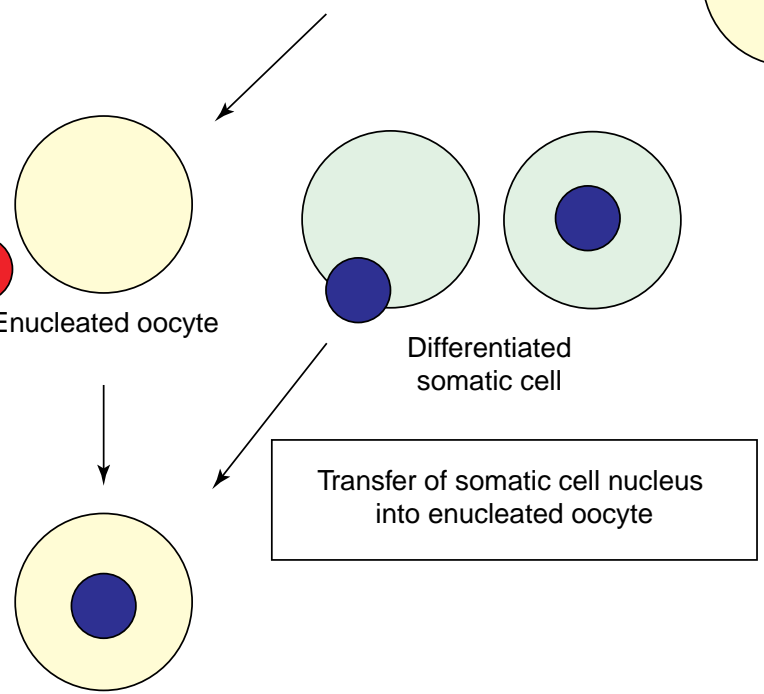

Reconstituted oocyte

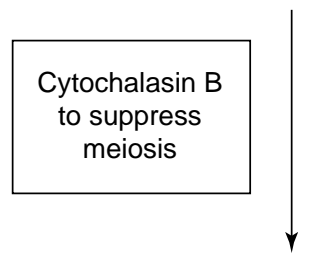

Reprogramming instructions to the somatic cell nucleus from oocyte cytoplasm

Embryonic development

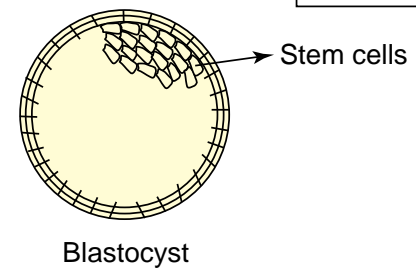

$\overline{T R E N D S ~ i n ~ B i o t e c h n o l o g y ~}$

Figure 1. Somatic cell nuclear transfer. Normal meiosis (a) is suppressed in the reconstituted oocyte by the presence of Cytochalasin B in the culture medium. It is instead

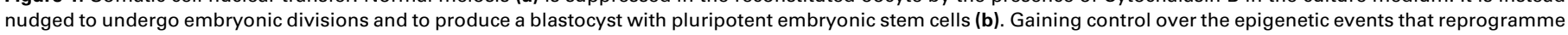
the reconstituted oocyte is one of the technological challenges in cloning. Abbreviations: M1, meiosis I; M2, meiosis II.

attempt meiosis but then aborts (apomeiosis) and undergoes three mitotic divisions to produce an unreduced FG (diplospory), or a diploid nucellar cell other than the MMC might undergo three mitotic divisions to produce an unreduced FG (apospory). In the second pathway, a nucellar cell can transdifferentiate directly into an embryo (adventitious embryony) [4] (Figure 2).

The in vivo potential for both transgenerational epigenetic inheritance through meiosis and epigenetic inheritance through mitotic divisions of the transdifferentiated embryo have conferred a mammalian stem cell- like developmental plasticity on the nucellar cells. These features have also raised hopes of an 'agronomic asexual revolution' in which clones can be produced through seeds if sexual crop plants can be genetically empowered with apomictic traits [5].

\section{Epigenetics and cell fate Epigenetic regulation}

Changes in gene expression patterns without corresponding changes in the DNA sequence - namely, epigenetics depends on the stable, heritable marking of DNA or 


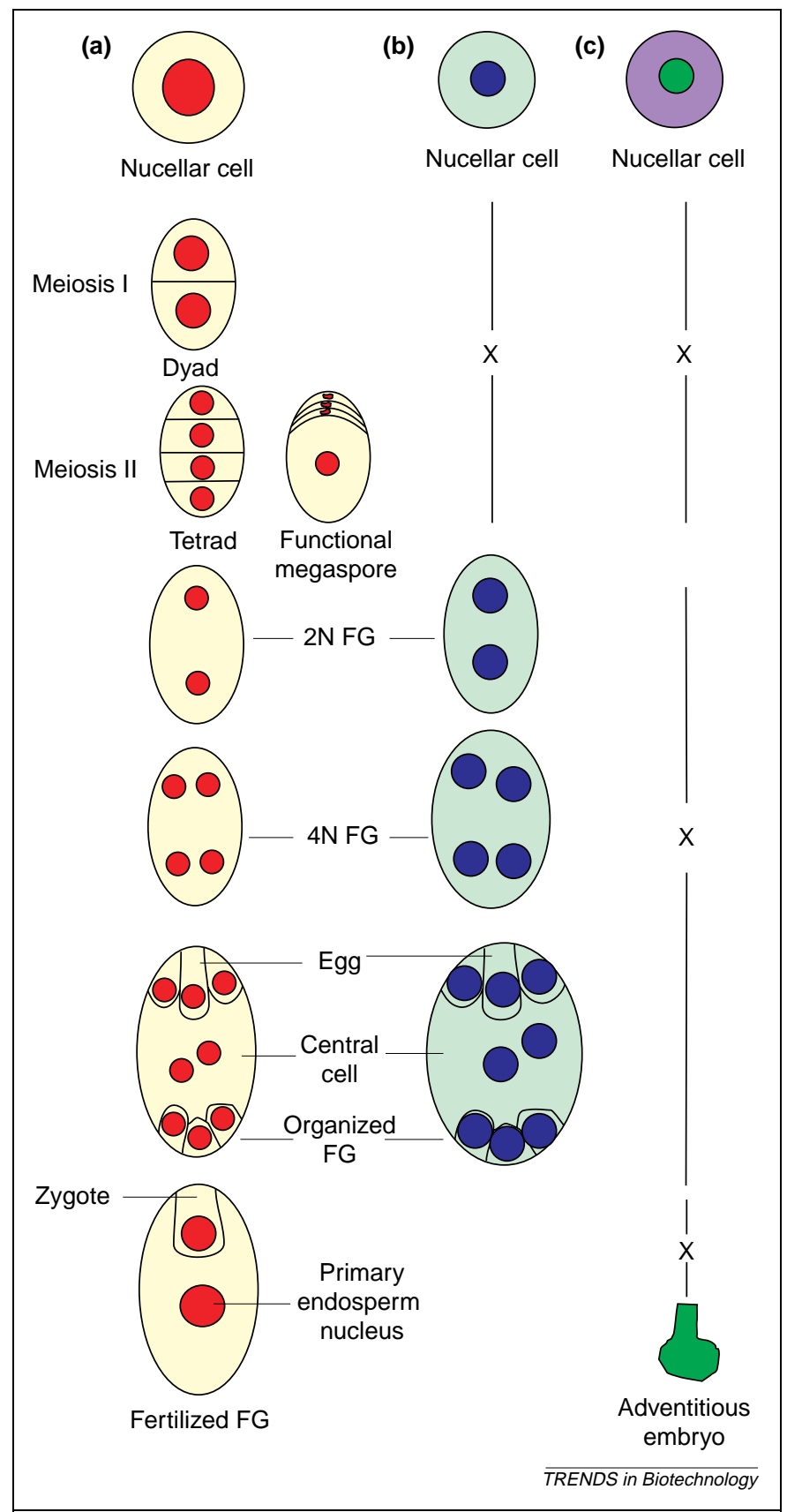

Figure 2. Developmental pathways in the nucellus. Nucellar cells can launch three different developmental pathways: (a) a sexual pathway, (b) a gametophytic apomixis (apospory/diplospory) and, (c), a sporophytic apomixis. Understanding the epigenetic mechanisms by which these three pathways are constructed by the nucellar cells holds the key to future apomixis-based research. Abbreviations: FG, female gametophyte.

chromatin. In eukaryotes, epigenetic regulation often involves the methylation of DNA, particularly that of cytosine residues. Failure to transmit faithfully a methylated or unmethylated state (locus) of cytosine can lead to altered phenotypes in plants and animals [6].

Theise and Wilmut [7] point out that, during animal development, cytosine residues of the newly formed DNA strands can be left unmethylated by a passive process for physiological reasons. In plants, methyltransferase 1 maintains DNA methylation on cytosine residues, whereas chromomethylase 3 targets other sites $[8,9]$. In addition, imprinting in Arabidopsis differs from that in mammals, where the methylation of specific DNA sequences in intergenic regions of up to $100 \mathrm{~kb}$ (imprinting control centres) regulates the expression of a group of genes [10]. In mammals, DNA in the germline cells is globally demethylated to erase the earlier imprints, and new ones are established during gametogenesis.

Box 1 provides a general account of developmental plasticity in animal cells. No such global demethylation has been detected in plants [11]. So to what extent are these comparisons relevant to the different developmental pathways in the nucellus?

\section{Developmental pathways in the nucellus}

Hierarchical cell lineage is a consequence of progressive restrictions imposed on gene expression patterns in differentiating cells. Such gene repression has to be overcome if a terminally differentiated cell has to jumpstart development or to transdifferentiate. For such developmental plasticity to be established conclusively, at least two criteria must be satisfied: first, cells must have proven clonality; and second, the contribution of the individual cells rather than the tissue must be established [12]. Although adventitious embryony - particularly, for example, in Citrus species, where up to 80 adventitious embryo initials per ovule are known [13] - points to the plasticity of nucellar cells, the apospory and diplospory pathways, which are edited versions of the normal sexual pathway, point to the developmental heterogeneity of nucellar cells.

There seem to be three epigenetic states in nucellar cells that facilitate the manifestation of differences in the developmental pathways: one that starts with meiosis followed by the formation of a FG with a haploid egg; a second that bypasses meiosis and forms an unreduced FG with a diploid egg; and a third that transdifferentiates a nucellar cell directly into an adventitious embryo by bypassing the sexual pathway (Figure 2). Considering that some basic requirements of transcription must be met for de novo protein production (Box 2), the challenge before us is to interpret how the nucellar cells construct different molecular platforms in vivo and launch each of the three pathways, which seem to be totally independent and mutually exclusive of each other.

\section{Elucidating the genetic basis of apomixis}

Cloning by SCNT relies on manipulating the fate of the stem cell or reconstituted oocyte by invoking epigenetic mechanisms under in vitro conditions. By contrast, plant scientists consider that a positive and inheritable genetic programme controls apomixis and have relied on classical breeding experiments to transfer apomixis genes to sexual crop plants [14]. Such an approach would greatly simplify breeding schemes and enable the fixation of any genotype including those of the $F_{1}$ hybrids. Although elegant in theory, practical applications are proving challenging because the genetic basis of apomixis remains elusive.

However, apomixis is beginning to be viewed as a deregulation of the sexual process in space and time, leading to putative changes in cell fate. It is thought that apomixis might not be controlled by mutant alleles but rather by epigenetic changes in gene regulation [15]. 


\section{Box 1. Developmental potential, plasticity and cell fate}

It was previously accepted that once a cell is terminally differentiated, it cannot be programmed to assume another fate. Somatic cell nuclear transfer (SCNT) has now shown that under appropriate in vitro conditions cellular totipotency can be restored (Figure 1). However, clear explanations of both the scientific issues and the related applications are necessary to exploit the full potential of this phenomenon.

The great promise of stem cells in human welfare lies in their capacity to self-renew and to assume many fates depending on their microenvironment or 'stem cell niche' [37]. The developmental potential of a (stem) cell is the sum total of all of the fates that it is capable of generating [38]. A stem cell is classified according to the number of different types of cell to which it can give rise: totipotent, pluripotent, multipotent, oligopotent and unipotent [39]. The zygote and the early embryonic stem cells (ESCs) are totipotent; by contrast, the germline stem cells are unipotent, giving rise to either male or female gametes. The haematopoietic stem cells are the bestcharacterized pluripotent stem cells; located in the bone marrow of mice and humans, they generate the dozen or more cell types of the blood [40].

Unlike a pluripotent ESC, an adult stem cell (ASC) or 'progenitor' cell is dedicated to a particular developmental pathway and is committed to generate a specific cell lineage (lineage commitment). Plasticity or lineage conversion describes the ability of an ASC to switch cell fate and to generate unexpected cell types [41]. There are many explanations for plasticity: (i) a change in the microenvironment or niche might cause a pluripotent or multipotent stem cell to respond to a new extracellular environment and to switch cell fate; (ii) owing to cell-cell fusion, the nucleus of one cell type might be reprogrammed by the cytoplasm of the other cell type [39]; and (iii) under some circumstances ASCs might cross the lineage boundary and 'transdifferentiate' into a type of new cell. Transdifferentiation describes the conversion of a cell of one tissue lineage into a cell of a completely different lineage. Spontaneous cell fusion, which mimics a transdifferentiation event, involves nuclear reprogramming and therefore is functionally equivalent to nuclear transfer [42]. Transdifferentiation is also considered to be physiologically equivalent to metaplasia, a pathological condition in which the abnormal conversion of one cell type into another can occur [43]. To establish true cell plasticity, therefore, stringent parameters for the proven clonality of the cell in question and the generality of the event in several models are needed [39].

Development, along with other cellular changes in state, can be conceived as the sequential adoption of stable, epigenetically determined patterns of gene expression [44]. Cloning by SCNT is basically a developmental problem in which the epigenetic regulation of cell fate is a fundamental issue: gene expression and presumably epigenetic modifications must be reprogrammed in the reconstituted oocyte to convert it to the early embryonic state [45]. A fast, programmed proteolysis, particularly in the ubiquitin-proteasome pathway, seems to be central to nuclear reprogramming after SCNT [46]. Thus, both in the reconstituted oocyte and in nucellar cells, whatever the changes in the epigenetic state, some basic requirements of transcription (Box 2) must be met for the de novo production of proteins.

So far, clones derived from SCNT have been generated in eight animal species; most of them have shown some abnormalities because of defects in the epigenetic reprogramming of the reconstituted oocyte [30]. The preparation of cytoplasts is a crucial step because these cells have to retain the ability to reprogramme the transferred nucleus to produce an embryo. Current micromanipulation skills therefore require simplification to achieve high success rates in cloning [47].

Mogie [16] has also pointed out that 'epialleles' behave like mutations. At present, little is known about the molecular switch that pushes the individual nucellar cells towards either the sexual pathway or the different apomictic pathways. The success of the current biotechnological initiatives that use apomixis therefore ultimately depends on resolution of the genetics-epigenetics (or mutationsepimutations) puzzle by shifting the focus of research from the nucellus as a tissue to individual nucellar cells.

Novel screening methods, such as laser capture microscopy combined with highly sensitive dissection procedures, will need to be developed to analyse the DNA and chromatin status of target loci in plant cells [17]. In addition, noninvasive techniques for tracking the development of individual nucellar cells in vivo will be needed to identify the precise molecular mechanisms involved. A hairpin-bisulfite polymerase chain reaction method that can detect methylation symmetry between complementary strands of individual DNA molecules [6], if suitably modified for plant tissues, could illuminate the situation. Monitoring single-cell transcription profiles by digital fluorescence microscopy, whereby the nucleus of each cell is tracked by oligonucleotide probes that colourcode the transcription profiles [18], also holds enormous promise in this regard.

In combination with sequence information from the model plants Arabidopsis and Zea mays, the advent of techniques that determine the transcription profiles of cells and organisms [19] and antisense technologies that can inhibit the expression of a specific target gene in a sequence-specific manner [20] could provide the necessary platform for exploring the proteomes of individual plant cells. The availability of a wide range of fluorescent probes [21,22] and advanced biological imaging techniques [23] might further the identification of functional cellular variation in an otherwise morphologically homogeneous nucellar tissue. A combination of high-throughput techniques for investigating the proteome (expressed protein component of the cell) is currently being developed under the concept of a 'molecular scanner' [24].

\section{Similarities between a reconstituted oocyte and a nucellar cell}

Although the developmental fates of a reconstituted oocyte and nucellar cell both seem to be moulded by epigenetic reprogramming, and both cell types are capable of returning to totipotency and producing an embryo directly, at present there is no evidence that they share similar or even remotely related pathways, particularly in view of the divergence of plants and animals in evolutionary history.

There are two alternative views to explain such functional similarities in the development of unrelated organisms. When homology is applied to genes - though functional homology is not true genetic homology [25] the concepts of paralogy (relationships owing to gene duplication) and orthology (genes originating from speciation) need to be considered. Orthologs take over the functions of the precursor gene in the species of origin and tend to be conserved, whereas paralogs have redundant function and are either lost or diverge in function [26]. However, the homology between the Drosophila gene piwi, which controls germline stem cells, and the Arabidopsis gene zwille, which controls stem cells in shoot meristems, is taken as evidence for a common origin of 'stemness' in 


\section{Box 2. Genome, epigenome and transcription}

All cells of an organism contain the same genetic information (genome), but additional epigenetic information, in the form of modifications to the DNA and to the chromatin-associated proteins (epigenome), decide the fate and functions of a cell. Cellular identity is a consequence of both the genes that are expressed and those that are not expressed in a chromatin context. The genome of a differentiating cell undergoes physical epigenetic changes. A dynamic interaction between the nucleus and the cytoplasm prevents inappropriate gene expression [48].

Nucleosomes are recognized as highly dynamic units of chromatin through which the eukaryotic genomes can be regulated epigenetically with heritable consequences [49]. The basic repeating unit of chromatin, the nucleosome, is typically composed of an octamer of the four core histones $(\mathrm{H} 2 \mathrm{~A}, \mathrm{H} 2 \mathrm{~B}, \mathrm{H} 3$ and $\mathrm{H} 4)$ and 146 base pairs of DNA wrapped around the histone. Numerous modifications to the nucleosome, involving both DNA and the histones, regulate genomic functions. In addition to DNA methylation, covalent modifications of the histones include acetylation of lysines, methylation of lysines and arginines, phosphorylation of serines and threonines, ubiquitination of lysines, sumoylation of lysines, and ADP-ribosylation of glutamic acid residues [50]. These epigenetic markers must be precisely copied after DNA replication to lead to heritable changes in chromatin structure. The net results of the epigenetic modifications can be transmitted to offspring during gametogenesis (transgenerational epigenetic inheritance) and through mitotic epigenetic inheritance, which helps to maintain either cellular memory during clonal divisions or cellular differentiation [51]. The epigenetic imprints acquired by the somatic cells during differentiation are not transferred to offspring because they are completely erased during gametogenesis [52].

The genomic (parental) imprints established during gametogenesis

both plants and animals [27]. It is not known whether piwi and zwille can replace each other in cellular function.

\section{Cytoplasm directs nuclear function}

Selectivity of gene expression depends on the ability of transcription factors to access the genome in time and space. Recent studies have highlighted some interesting parallels between gene expression in the reconstituted oocyte and that in plant somatic embryogenesis. Two principles have emerged from the first half-century of nuclear transplantation: the conservation of the genome during cell differentiation and the ability of the cell cytoplasm to reprogramme gene activity, and therefore to redirect cell differentiation [28].

Both amphibian and mammalian nuclear transfer experiments have shown that there is a strong positive correlation between the gene expression patterns of a reconstituted oocyte and those of a fertilized embryo [28]. The nuclear transcription factors Oct4, Sox2, FoxD3, Stat3 and Nanog have some ability to restore embryoniclike plasticity to adult stem cells in mouse [29]. The importance of the oocyte cytoplasm in the epigenetic programming of the reconstituted oocyte has prompted the development of in vitro systems that prime the donor cells and their chromatin by prior exposure to remodelling factors that are normally present in the oocyte cytoplasm in order to achieve high rates of totipotency of reconstituted oocytes [30].

Viewed from these angles, the behaviour of individual nucellar cells needs to be understood in order to explain epigenetic regulation of gene expression during developmental transitions. For example, the LEAFYCOTYLEDON1 (LEC1) [31], LEC2 [32], BABY BOOM [33], SOMATIC are maintained after fertilization as the chromosomes are duplicated and segregated in the developing organism. In the new organism (offspring), however, these parental imprints are completely erased and new ones are established in a parent-of-origin manner. Parental genomes therefore show epigenetic asymmetry at fertilization that persists throughout life, whereas the epigenetic state acquired for cell identity during development might change depending on the developmental context [53]. Thus, genomes of differentiated somatic cells carry both parental imprints and epigenetic marks acquired through the differentiation process. During SCNT, the parental imprints must be protected from the reprogramming of the somatic nucleus that is introduced into the reconstituted oocyte [54].

An early step that initiates gene expression is the binding of a transcription factor to its target DNA site. A transcription factor can bind to a site that is not blocked by a nucleosome [55]. Thereafter, the formation of a pre-initiation complex at the right time and at the right promoter is a prerequisite to execute the correct gene expression programme of mRNA synthesis [56]. Nucleosomes unfold completely at transcriptionally active promoters [57]. Nucleosome remodelling can either facilitate or repress mRNA synthesis. Many types of chromatin modifying factor are conserved between plants and animals, although those in plants might have some novel features [58]. Transcription is carried out by three RNA polymerases: RNA polymerase I (Pol I), which transcribes rRNA genes; RNA Pol II, which transcribes protein-encoding genes and some small nuclear RNAs (snRNAs); and Pol III, which transcribes most of the small RNAs (snRNAs and tRNAs) [59]. The current technologies available for interrogating the transcriptome have been recently reviewed by Ruan et al. [19].

EMBRYOGENESIS RECEPTOR KINASE, which is expressed in carrot embryogenic cultures that form somatic embryos [34], and WUSCHEL [35] gene products can induce embryo formation in vegetative cells. How are these genes regulated in the nucellar cell during the in vivo transdifferentiation of an adventitious embryo? Although the initiation of zygotic, apomictic and in vitro embryogenesis is activated by different signals and often begins with different starting tissues, each of these processes can converge on the same signalling pathway at a very early stage [33].

Similar questions should be addressed with regard to the signals that direct the nucellar cell to sidestep meiosis and to jumpstart development to form a diploid apomictic FG. Nucellar cell transdifferentiation also needs to be understood both against the backdrop of the potential reversibility of plant cell fate even outside the nucellus and in a much wider developmental context namely, homogeneity in the differentiation state of the nucellar cells.

\section{Future perspectives}

Genetic manipulation of apomixis for crop improvement is an extremely attractive proposal because it could facilitate the production of clones through seeds, thereby helping to address the genomic imbalance that is often encountered in F1 hybrids. Recently, de novo engineering of genomic imprints - which control the inheritance of apomeiosis, parthenogenesis and endosperm development - have also been proposed as desirable goals for the development of future apomixis technologies [36]. Theoretical issues of bio-safety and intellectual property management regarding the impact of 'infectious apomixis' [i.e. the spread of 


\section{Box 3. Outstanding questions}

- The most fundamental question in the future of apomixis-based biotechnology is whether the different apomictic pathways in the nucellus are under genetic control or under an epigenetic influence that affects the clonality of the nucellar cell population.

- Fifty years of nuclear transfer experiments have shown that nuclear DNA does what it is ordered to do by the cell cytoplasm. So how do nucellar cells programme their genome to construct the molecular platforms to launch three different and independent developmental pathways? The answer to this question will be crucial for a holistic understanding of the basic biology of developmental programming and reprogramming in eukaryotes.

- How does Cytochalasin B suppress meiosis in the reconstituted oocyte and what are the corresponding molecular events in the nucellar cell that avoids meiosis and develops to produce an unreduced (apomictic) female gametophyte?

apomixis by a dominant transgene (apomixis supergene) to a related outcrossing species and the consequent ecological risks due to possible displacement of the sexual siblings] are already being debated [36]. The immense popularity of these concepts has perhaps made it very difficult to look for an alternative, even though a genetic basis for apomixis has been elusive so far. But a reassessment of the classical genetic approaches, on the basis of the 'gene as hereditary unit', seems to be on the cards in view of recent developments in mammalian stem cell biology.

It has been shown in mammals that both transdifferentiation, which can return a differentiated cell to a totipotent state, and lineage conversion or plasticity, which generates cells of totally unexpected fate, are under epigenetic regulation. The idea that apomixis might be also under epigenetic regulation has emerged recently [15]. Indeed, the presence of both sexual and apomictic pathways in the same plant, sometimes in the same ovule, points more towards an epigenetic influence over the clonality of the nucellar cell population.

Given the lack of crucial details concerning apomictic regulation (Box 3 ), and the highly conserved nature of the pathways involved (Figure 2), the field is now ripe for applying the full power of functional genomics to explore and to define the molecular mechanisms driving the developmental pathways in the nucellus and to bring them into clear focus. Notwithstanding the exciting opportunities, it is a long way to go in basic research before the necessary scientific base will be established to take full advantage of the potential of nucellar cells. We can progress as we learn and learn as we progress.

\section{Acknowledgements}

This article is dedicated to apomixis researchers who are striving to usher in an asexual revolution in crop production.

\section{References}

1 Hubner, K. et al. (2003) Derivation of oocytes from mouse embryonic stem cells. Science 300, 1251-1256

2 Geijsen, N. et al. (2004) Derivation of embryonic germ cells and male gametes from embryonic stem cells. Nature 427, 148-154

3 Wilmut, I. et al. (2002) Somatic cell nuclear transfer. Nature 419, 583-587

4 Asker, S.E. and Jerling, L. (1992) Apomixis in Plants, CRC Press

5 Vielle-Calzada, J-P. et al. (1996) Apomixis - the asexual revolution. Science 274, 1322-1323
6 Laird, C.D. et al. (2004) Hairpin-bisulfite PCR: assessing epigenetic methylation patterns on complementary strands of individual DNA molecules. Proc. Natl. Acad. Sci. U. S. A. 101, 204-209

7 Theise, N.D. and Wilmut, I. (2003) Cell plasticity: flexible arrangement. Nature 425, 21

8 Finnegan, E.J. et al. (1996) Reduced DNA methylation in Arabidopsis thaliana results in abnormal plant development. Proc. Natl. Acad. Sci. U. S. A. 93, 8449-8454

9 Lindroth, A.M. et al. (2001) Requirement of CHROMOMETHYLASE3 for maintenance of CpXpG methylation. Science 292, 2077-2080

10 Berger, F. (2004) Imprinting - a green variation. Science 303, $483-485$

11 Saze, H. et al. (2003) Maintenance of CpG methylation is essential for epigenetic inheritance during plant gametogenesis. Nat. Genet. 34, $65-69$

12 Orkin, S.H. and Zon, L.I. (2002) Hematopoiesis and stem cells: plasticity versus developmental heterogeneity. Nat. Immunol. 3, 323-328

13 Rangan, T.S. and Rangaswamy, N.S. (1999) Nucellus - a unique embryonic system. Phytomorphology 49, 337-376

14 Grossniklaus, U. et al. (2001) How to avoid sex? The genetic control of gametophytic apomixis. Plant Cell 13, 1491-1498

15 Koltunow, A.M. and Grossniklaus, U. (2003) Apomixis: a developmental perspective. Annu. Rev. Plant Biol. 54, 547-574

16 Mogie, M. (1992) The Evolution of Asexual Reproduction in Plants, Chapman \& Hall

17 Steimer, A. et al. (2003) Epigenetic control of plant development: new layers of complexity. Curr. Opin. Plant Biol. 7, 11-19

18 Levsky, J.M. (2003) Simple single cells. Science 302, 585

19 Ruan, Y. et al. (2004) Interrogating the transcriptome. Trends Biotechnol. 22, 23-30

20 Kurreck, J. (2003) Antisense technologies: improvement through novel chemical modifications. Eur. J. Biochem. 270, 1628-1644

21 Zhang, J. et al. (2002) Creating new fluorescent probes for cell biology. Nat. Rev. Mol. Cell Biol. 3, 906-918

22 Miyawaki, A. (2003) Visualization of the spatial and temporal dynamics of intracellular signaling. Dev. Cell 4, 295-305

23 Hurtley, S.M. and Helmuth, L., eds (2003) Special issue on biological imaging. Science 300, 75-102

24 Binz, P-A. et al. (2004) The molecular scanner: concept and developments. Curr. Opin. Biotechnol. 15, 17-23

25 Abouheif, E. et al. (1997) Homology and developmental genes. Trends Genet. 13, 432-433

26 Theissen, G. (2002) Secret life of genes. Nature 415, 741

27 Benfey, P.N. (1999) Stem cells: a tale of two kingdoms. Curr. Biol. 9, R171-R172

28 Gurdon, J.B. and Byrne, A.B. (2003) The first half-century of nuclear transplantation. Proc. Natl. Acad. Sci. U. S. A. 100, 8048-8052

29 Cavaleri, F. and Scholer, H.R. (2003) Nanog: a new recruit to the embryonic stem cell orchestra. Cell 113, 551-552

30 Alberio, R. and Campbell, K.H.S. (2003) Induction of transcription in bovine fetal fibroblasts exposed to Xenopus laevis egg extracts. Theriogenology 59, 318

31 Lotan, T. et al. (1998) Arabidopsis LEAFY COTYLEDON 1 is sufficient to induce embryo development in vegetative cells. Cell 93, 1195-1205

32 Stone, S.L. et al. (2001) LEAFY COTYLEDON2 encodes a B3 domain transcription factor that induces embryo development. Proc. Natl. Acad. Sci. U. S. A. 98, 11806-11811

33 Boutilier, K. et al. (2002) Ectopic expression of BABY BOOM triggers a conversion from vegetative to embryonic growth. Plant Cell 14, 1737-1749

34 Schmidt, E.D. et al. (1997) A leucine-rich repeat containing receptorlike kinase marks somatic plant cells competent to form embryos. Development 124, 2049-2062

35 Zuo, J. et al. (2002) The WUSCHEL gene promotes vegetative to embryonic transition in Arabidopsis. Plant J. 30, 349-359

36 Spillane, C. et al. (2004) Apomixis technology development - virgin births in farmers' fields? Nat. Biotechnol. 22, 687-691

37 Fuchs, E. et al. (2004) Socializing with the neighbours: stem cells and their niche. Cell 116, 769-778

38 Temple, S. (2001) Stem cell plasticity - building the brain of our dreams. Nat. Rev. Neurosci. 2, 513-520 
39 Wagers, A.J. and Weissman, I.L. (2004) Plasticity of adult stem cells. Cell 116, 639-648

40 Kluger, Y. et al. (2004) Lineage specificity of gene expression patterns. Proc. Natl. Acad. Sci. U. S. A. 101, 6508-6513

41 Holden, C. and Vogel, G. (2002) Plasticity: a time for reappraisal? Science 296, 2126-2129

42 Raff, M. (2003) Adult stem cell plasticity: fact or artifact? Annu. Rev. Cell Dev. Biol. 19, 1-22

43 Rasco, J.E.J. (2003) Transdifferentiation: an attractive concept in search of evidence. Pathology 35, 5

44 Voth, W.P. and Stillman, D.J. (2003) Changes in developmental statedemolish the old to construct the new. Genes Dev. 17, 2201-2204

45 Wrenzycki, C. (2003) Epigenetic reprogramming in early embryonic development: effects of in vitro production and somatic nuclear transfer. Reprod. Biomed. Online 7, 649-656

46 Sutovsky, P. and Prather, R.S. (2004) Nuclear remodeling after SCNT: a contractor's nightmare. Trends Biotechnol. 22, 205-208

47 Fulka, J., Jr. et al. (2004) Nucleus transfer in mammals: noninvasive approaches for the preparation of cytoplasts. Trends Biotechnol. 22, 279-283

48 Colman, A. (2002) Turning back the developmental clock. Nat. Biotechnol. 20, 348-349

49 Khorasanizadeh, S. (2004) The nucleosome: from genomic organization to genomic regulation. Cell 116, 259-272
50 Bannister, A.J. et al. (2002) Histone methylation: dynamic or static? Cell 109, 801-806

51 Rakyan, V. and Whitelaw, E. (2003) Transgenerational epigenetic inheritance. Curr. Biol. 13, R6-R8

52 Shi, W. et al. (2003) Epigenetic reprogramming in mammalian nuclear transfer. Differentiation 71, 91-113

53 Surani, M.A. (2001) Reprogramming of genome function through epigenetic inheritance. Nature 414, 122-128

54 Reik, W. and Walter, J. (2001) Genomic imprinting: parental influence on the genome. Nat. Rev. Genet. 2, 21-32

55 Ahmad, K. and Henikoff, S. (2002) Epigenetic consequences of nucleosome dynamics. Cell 111, 281-284

56 Hochheimer, A. and Tjian, R. (2003) Diversified transcription initiation complexes expand promoter selectivity and tissue specific gene expression. Genes Dev. 17, 1309-1320

57 Boeger, H. et al. (2003) Nucleosomes unfold completely at a transcriptionally active promoter. Mol. Cell 11, 1587-1598

58 Wagner, D. (2003) Chromatin regulation of plant development. Curr. Opin. Plant Biol. 6, 20-28

59 Zhou, P. and Ly, D.H. (2003) Embryonic stem cells: a perfect marriage between gene regulation and regenerative medicine. Curr. Top. Med. Chem. 3, 725-737

\section{Getting animated with parasites!}

Interested in the molecular cell biology of host-parasite interactions? Then take a look at the online animations produced by Trends in Parasitology, one of our companion TRENDS journals. The pictures below are snapshots from two of our collection of animations revealing the latest advances in understanding parasite life cycles. Check them out today!

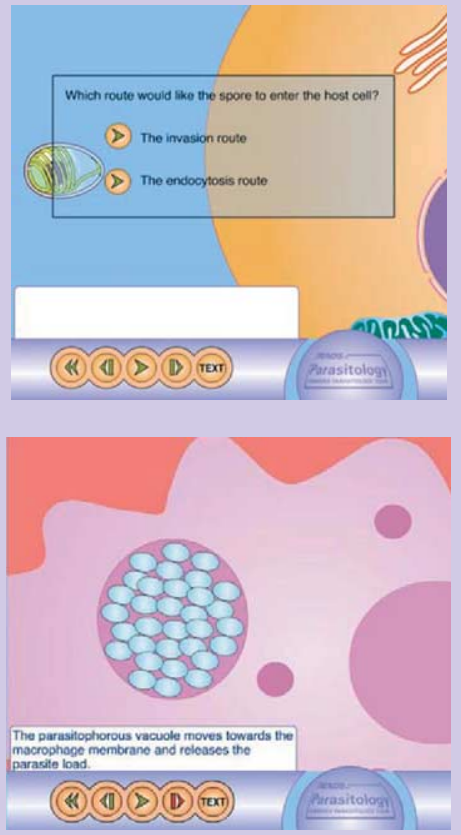

Microsporidia: how can they invade other cells?

By C. Franzen [(2004) Trends Parasitol. 20, 10.1016/j.pt.2004.04.009] http://archive.bmn.com/supp/part/franzen.html

Interaction of Leishmania with the host macrophage

By E. Handman and D.V.R. Bullen [(2002) Trends Parasitol. 18, 332-334] http://archive.bmn.com/supp/part/swf012.html 